\title{
Re: Genomic Markers in Prostate Cancer Decision Making
}

\author{
Cucchiara V1, Cooperberg MR2, Dall'Era M11, Lin DW3, Montorsi F4, Schalken JA5, Evans CP1 \\ 1 University of California Faculty of Medicine, Department of Urology, Sacramento, CA, USA \\ 2University of California Faculty of Medicine, Departments of Urology and Epidemiology \& Biostatistics, San Francisco, CA, USA \\ 3University of Washington Faculty of Medicine, Department of Urology, Seattle, WA, USA \\ 4 Vita-Salute University San Raffaele Scientific Institute, Urological Research Institute, Department of Urology, Milan, Italy \\ ${ }^{5}$ Radboud University Medical Center, Department of Urology, Nijmegen, The Netherlands \\ Eur Urol 2018;73:572-582. doi: 10.1016/j.eururo.2017.10.036. Epub 2017 Nov 10.
}

\section{EDITORIAL COMMENT}

Prostate cancer ( $\mathrm{PCa}$ ) is the most common urological cancer in men and prostate-specific antigen remains one of the most useful biomarkers for the diagnosis and treatment of this disease. However, it has limited specificity and there is a need for more sensitive and specific biomarkers. In this context, after the extraordinary development in molecular and genomic techniques, new biomarkers have been described. These assays have been classified as the urine-based, blood-based and tissue-based methods. SelectMDx, MiProstate Score, and ExoDx can be useful in identification of patients who may benefit from prostate biopsy. PCA3 and ConfirmMDx predict the outcome of subsequent biopsy in men with previous negative pathological findings. Decipher, Oncotype DX, and Prolaris are commercially available tools and can be used for PCa risk stratification and identifying men at the highest risk of adverse outcome. Furthermore, some markers, such as androgen receptor splice variant 7 (AR-V7) expression and mutations in DNA mismatch repair genes, may help select treatment in castration-resistant PCa. AR-V7 expression predicts resistance to abiraterone/enzalutamide, while poly polymerase- 1 inhibitor and platinum-based chemotherapy could be indicated in metastatic patients who are carriers of mutations in DNA mismatch repair genes. Introduction genomic biomarkers have improved the detection, prognosis, and risk evaluation of PCa. In the near future, we may use many biomarkers for diagnosis, follow-up and treatment of PCa.

Fehmi Narter, MD, PhD

${ }^{\oplus}$ Copyright 2019 by the Association of Urological Surgery / Journal of Urological Surgery published by Galenos Publishing House. 